Table 2 Tests for Helicobacter pylori

\begin{tabular}{llll}
\hline Test & $\begin{array}{l}\text { Sensitivity } \\
(\%)\end{array}$ & $\begin{array}{l}\text { Specificity } \\
(\%)\end{array}$ & NND \\
\hline Chronic inflamation & 100 & 66.3 & 1.51 \\
Acute inflamation & 86.7 & 93.7 & 1.24 \\
Staining H pylori & 93.1 & 99.4 & 1.08 \\
CLO test & 89.6 & 100 & 1.12 \\
Urea breath test & 90.2 & 95.3 & 1.16 \\
Serum IgG antibodies & 91.3 & 91.6 & 1.21 \\
Serum IgA antibodies & 71.1 & 89.8 & 1.64 \\
\hline
\end{tabular}

presence of disease and gives a ready comparison between tests (table 2).

However, if cost data are available it is possible to ask questions about the value of biopsy and CLO test at, say, $£ 170$ versus an antibody test at $£ 7$ when the difference in NND is only 0.09 . From this it may be calculated each positive diagnosis costs $£ 190.40$ with the former test and $£ 8.50$ with the latter. Where there are large differences in costs, the option of using the cheaper as a front line test and the more expensive as the back up may be considered. It is also possible to allow for other "quality" factors such as waiting times and patient acceptability, ${ }^{10}$ both of which favour the non-invasive test with the only advantage of the invasive route being that Koch's postulates are more closely met and the possibility that "classic" peptic ulcer symptoms might mask an operable malignancy in a younger person.

\section{Concluding comments}

The concepts of evidence based practice are a stimulus to pathologists to:
- use criteria based on research evidence in their diagnostic work;

- apply research evidence in interpretation of laboratory data;

- find new ways of presenting findings to assist their fuller interpretation;

- use cost and NND to help develop rational approaches to testing strategies; and

- help clinical colleagues use research evidence with pathology diagnostic findings more effectively.

GIFFORD BATSTONE

Professor, Postgraduate Medical Education,

University of Nottingham

1 Fraser CG. Interpretation of clinical laboratory data. Oxford: Blackwell Scientific Publications, 1986.

2 Antman EM, Lau J, Kupelnick B, Mosteller F, Chalmers TC. A comparison of results of meta-analysis of randomized control trials and the recommendations of clinical experts. $\mathscr{f} A M A$ 1992;268:240-8.

3 Jaeschke R, Guyatt G, Sackett DL. User's guide to the medical literature. III. How to use an article about a diagnostic test. $\mathcal{F} A M A$ 1994;271:389-91; 703-7.

4 McKibbon KA, Walker CJ. How to harness MEDLINE for diagnostic problems. ACP Journal Club. Sep-Oct. Ann Intern Med 1994;121(Suppl 2):A10.

5 Sackett DL, Hayne RB, Guyatt GH, Tugwell P. Clinical epidemiology; a basic science for clinical medicine. Boston: Little, Brown and Company, 1991:1039.

6 Anonymous. How good is that test-using results. Oxford: Bandolier, 1996;(3):6-8.

7 Lachs MS, Nachamkin I, Edelstein PH, Goldman J, Feinstein AR, Schwartz JS. Spectrum bias in the evaluation of diagnostic tests: lessons from the rapid dipstick test for urinary tract infection. Ann Intern Med 1992;117: $135-40$.

8 Anonymous. Diagnostic strategies. Bandolier 1996;(29):2-3.

9 Cutler AF, Havstad S, Ma CK, Blaser MJ, Perez-Perez GI, Schubert TT Accuracy of invasive and non-invasive tests to diagnose Helicobacter pylori infection. Gastroenterology 1995;109:136-41.

10 Guyatt GH, Sackett DL, Sinclair JC, Hayward R, Cook DJ, Cook RJ. User's guide to the medical literature. IX. A method for grading health care recommendations. Evidence-based Medicine Working Group. $\mathfrak{F} A M A$ 1995;274: $1800-4$

\title{
Benefits and limitations of computerised laboratory data
}

Computers have been an integral part of laboratory life for many years. Their value is self evident-without them many laboratory functions could simply not be done.

Computer functions are not always completely understood by those who operate them, and errors may not be evident or easily detectable. In medical laboratory diagnosis this places a burden on those "in charge" that has service, legal, and ethical consequences. Errors may extend from the quality and accuracy of data, through the adequacy of data storage and processing, and the form of their presentation to peripheral users, to ensuring the availability of reports to those who need them, ${ }^{1}$ as well as ensuring that they are not available to unauthorised users.

\section{Direct benefits}

Direct benefits encompass administrative elements such as accounting and ordering of material and equipment, as well as professional elements such as quality and extent of service. Much depends on the interests and imagination of the users and developers of the system. The integration of information systems at the Hadassah-University Hospitals in Jerusalem is a good example of microbiology, biochemistry, haematology, and pharmacy data being deployed together to provide relevant data for infectious disease consultants reviewing antimicrobial treatment in individual cases and tracking patients' movements between wards. Similar systems have been developed and applied elsewhere. ${ }^{2-4}$ Many would agree that selective reporting of antibiotic sensitivity results, ${ }^{5}$ easily achieved in a reasonable computerised system, has a contribution to make in promoting good treatment. "Flagging" selected pathogenic or drug resistant organisms ${ }^{5}$ allows the timely alert of clinicians and other professionals, such as infection control personnel. Built-in checks for inconsistencies in data can go a long way towards reducing the task of those scrutinising results before their issue. The internet and world wide web are also being explored for their potential in conformity of reporting practices ${ }^{6}$ and in developing more comprehensive clinical laboratory information systems. ${ }^{7}$

\section{Indirect benefits}

Indirect benefits may be no less important including monitoring trends of clinical or epidemiological problems, or even appropriateness and extent of laboratory use. ${ }^{8}$ A good system would expedite notification of communicable diseases to health authorities. ${ }^{9}$ Good in-house or commercially available systems will incorporate these capabilities, and more.

\section{Limitations}

LIMITATIONS INHERENTLY DETERMINED BY THE TYPE OF LABORATORY

While data captured on-line from automated tests can be stored and entered into patient records without any manual or subjective input from laboratory personnel, the situation in less automated settings, such as pathology or clinical 
microbiology laboratories, is vastly different. Here, systems have to deal with a lot of verbiage and subjectiveness that may be saved and retrieved in the form of coded comments, which are relatively easy to handle and exploit. Free text, which is often used, is the bane of anyone using the database for assessing laboratory operations or for clinical research. Even "simple" data can pose significant problems for analysis-anyone who has tried to estimate antibiotic resistance rates will have had to contend with the issue of excluding identical isolates from individual patients. Do we exclude identical isolates from any anatomical site, or only those from the same site? Is "identical" defined by antibiograms, biotypes, or molecular characterisation?

\section{LIMITATIONS INTRINSIC TO THE DESIGN AND CAPABILITIES OF} THE COMPUTER SYSTEM

If a clinical microbiology computer system does not provide refinements such as selective reporting of antibiotic sensitivities or periodic reports allowing monitoring of sensitivity patterns, the limitations are evident, but will not dramatically affect patient care. If, however, the absence of a system of data quality assurance, such as logical checks, allows inaccurate information to enter reports, the potential for negative effects on patient care might be significant.

\section{LIMITATIONS DETERMINED BY THE SOURCE AND NATURE OF} THE DATA

Any system involving manual data input will be susceptible to errors that are essentially undetectable. If an $S$ (sensitive) were entered erroneously instead of an $R$ (resistant) on a microbiology worksheet, the error could only be identified by a computerised check if a definable inconsistency arises - for example, proteus sensitive to colistin. In many other cases, the $S$ or $R$ might be equally acceptable, so the computer and the scrutineer, who must approve the report, will be unable to detect the error.

\section{Teamwork}

A multidisciplinary team approach to developing in-house systems or evaluating commercial systems for purchase is required if all potential drawbacks and inadequacies are to be minimised and all potential benefits realised. This team is equally important in assessing the performance of a computerised information system after its introduction. No single professional will be able to deal with all aspects, especially where there is integration with other clinical and administrative systems.

COLIN BLOCK

Head, Clinical Microbiology Unit,

Hadassah-University Hospital,

PO Box 12000, ferusalem il-91 120

Israel,

e-mail:block@hadassah.org.il

1 Block C, Laloum J, Rajs A, Stalnikowitz R, Shapiro M. Limitations of paperless on-line reporting of diagnostic bacteriology culture results. $\mathcal{f}$ Clin Pathol 1996;49:759-61.

2 Morrell R, Wasilauskas B, Winslow R. Personal computer-based expert system for quality assurance of antimicrobial therapy. Am F Hosp Pharm 1993; 50:2067-73.

3 Schentag J, Ballow CH, Fritz AL, Paladino JA, Williams JD, Cumbo TJ, et al. Changes in antimicrobial usage resulting from interactions among clinical pharmacy, the infectious disease division, and the microbiology laboratory. Diagn Microbiol Infect Dis 1993;16:255-64.

4 Michael PA. ROUNDS: a customizable HELP results review program for hospital staff physicians. Proc Annu Symp Comput Appl Med Care 1992:327-31.

5 Sharp SE. Effective reporting of susceptibility test results. Diagn Microbiol Infect Dis 1993;16:251-4.

6 Forrey AW, McDonald CJ, DeMoor G, Huff SM, Leavelle D, Leland D, et al. Logical observation identifier names and codes (LOINC) database: a public use set of codes and names for electronic reporting of clinical laboratory test results. Clin Chem 1996;42:81-90.

7 Willard KE, Hallgren JH, Sielaff B, Connelly DP. The deployment of a World Wide Web (W3) based medical information system. Proc Annu Symp Comput Appl Med Care 1995:771-5.

8 Studnicki J, Bradham DD, Marshburn J, Foulis PR, Straumfjord JV. A feedback system for reducing excessive laboratory tests. Arch Pathol Lab Med 1993;117:35-9.

9 Grant AD, Eke B. Application of information technology to the laboratory reporting of communicable diseases in England and Wales. Commun Dis Rep CDR Rev 1993;3:R75-8. 